\title{
Scaling relations and critical exponents for two dimensional two parameter maps
}

\author{
D. Stynes ${ }^{1}$, W.G. Hanan ${ }^{1}$, S. Pouryahya ${ }^{1}$, and D.M. Heffernan ${ }^{1,2, a}$ \\ 1 Department of Mathematical Physics, National University of Ireland Maynooth, Maynooth, Co. Kildare, Ireland \\ 2 School of Theoretical Physics, Dublin Institute for Advanced Studies, Dublin 4, Ireland
}

Received 3 November 2009 / Received in final form 15 July 2010

Published online 16 September 2010 - (C) EDP Sciences, Società Italiana di Fisica, Springer-Verlag 2010

\begin{abstract}
In this paper we calculate the critical scaling exponents describing the variation of both the positive Lyapunov exponent, $\lambda^{+}$, and the mean residence time, $\langle\tau\rangle$, near the second order phase transition critical point for dynamical systems experiencing crisis-induced intermittency. We study in detail 2-dimensional 2-parameter nonlinear quadratic mappings of the form: $X_{n+1}=f_{1}\left(X_{n}, Y_{n} ; A, B\right)$ and $Y_{n+1}=f_{2}\left(X_{n}, Y_{n} ; A, B\right)$ which contain in their parameter space $(A, B)$ a region where there is crisisinduced intermittent behaviour. Specifically, the Henon, the Mira 1, and Mira 2 maps are investigated in the vicinity of the crises. We show that near a critical point the following scaling relations hold: $\langle\tau\rangle \sim\left|A-A_{c}\right|^{-\gamma}$, $\left(\lambda^{+}-\lambda_{c}^{+}\right) \sim\left|A-A_{c}\right|^{\beta_{A}}$ and $\left(\lambda^{+}-\lambda_{c}^{+}\right) \sim\left|B-B_{c}\right|^{\beta_{B}}$. The subscript $c$ on a quantity denotes its value at the critical point. All these maps exhibit a chaos to chaos second order phase transition across the critical point. We find these scaling exponents satisfy the scaling relation $\gamma=\beta_{B}\left(\frac{1}{\beta_{A}}-1\right)$, which is analogous to Widom's scaling law. We find strong agreement between the scaling relationship and numerical results.
\end{abstract}

\section{Introduction}

The phenomenon of crisis-induced intermittency has been widely investigated [1-3] and many associated properties studied. The mechanisms which precipitate the occurrence of various crises are well understood [4] and numerical experiments have confirmed analytic predictions for many diverse chaotic systems. Crisis induced intermittency has been observed in a wide variety of systems including, recently, two dimensional viscous flow with non slip boundaries [5], the low dimensional limit of chaotic Alfven waves in space plasmas governed by the derivative Schrodinger equation [6], and two coupled logistic maps [7]. Of particular interest is the scaling of dynamical quantities close to the critical point after the occurrence of the crisis. In this post-crisis regime the dynamics describing the evolution of the system is intermittent, alternating between bursts of time spent in an enlarged phase space and periods spent on the attractor which existed before the crisis. Grebogi et al. [4] proposed a scaling for $\langle\tau\rangle$, the mean time a chaotic orbit spends in the region of the pre-crisis attractor. As a control parameter (which throughout we generically label as $A$ or $B$ ) is varied and the value of $\left|A-A_{c}\right|$ increases, it is found that $\langle\tau\rangle$ decreases according to the scaling relation $\langle\tau\rangle \sim\left|A-A_{c}\right|^{-\gamma}$ where $\gamma$ is the exponent describing the scaling of $\langle\tau\rangle$ with a parameter $A$. Close agreement has been found between the predic-

\footnotetext{
a e-mail: dmh@thphys.nuim.ie
}

tions of Grebogi et al. [4] and numerical simulations with various crises.

In this paper we use concepts from second order phase transitions in statistical physics to assist in attaining a deeper understanding of this dynamical phenomenon for two parameter maps. The maps that we study will be of the form:

$$
\begin{aligned}
X_{n+1} & =f_{1}\left(X_{n}, Y_{n} ; A, B\right) \\
Y_{n+1} & =f_{2}\left(X_{n}, Y_{n} ; A, B\right) .
\end{aligned}
$$

It will be shown that there is a transition from one chaos regime to another chaos regime via crisis which can be interpreted as a second-order phase transition. The following three specific maps, which contain such a crisis are studied:

(A) The Henon map. The Henon map is a non-linear two-dimensional mapping which has the following functional form

$$
\begin{aligned}
X_{n+1} & =1+Y_{n}-A X_{n}^{2} \\
Y_{n+1} & =B X_{n} .
\end{aligned}
$$

A crisis is observed to occur when the parameter $A$ falls in the following interval

$$
A_{c} \in[1.2716856034576725,1.2716856034576735]
$$

where $A_{c}$ denotes the critical value of the parameter $A$. The other parameter $B$ has the following value at the crisis: $B=0.3$ 
We have a chaos-chaos transition which is manifested by a change in the structure of the attractor as we go through the critical point. See Appendix A for further details on the map. All of the maps considered in this paper exhibit this property of structural change of the attractor through crisis.

(B) The First Mira map. The first Mira map [8] has the functional form

$$
\begin{aligned}
X_{n+1} & =Y_{n} \\
Y_{n+1} & =A Y_{n}-X_{n}^{2}+B .
\end{aligned}
$$

A crisis is observed to occur when the parameter, $B$, falls in the following interval

\section{$B_{c} \in[1.3000324223680495,1.3000324223680502]$}

where $B_{c}$ denotes the critical value of the parameter $B$. The other parameter has the following value at the crisis: $A=0.1$

See Appendix B for details of the map.

(C) The Second Mira map. The second Mira map [8] has the functional form

$$
\begin{aligned}
X_{n+1} & =A X_{n}+Y_{n} \\
Y_{n+1} & =X_{n}^{2}+B .
\end{aligned}
$$

A crisis is observed to occur when the parameter, $B$, falls in the following interval

$$
B_{c} \in[-2.05012267960083,-2.05012267960082]
$$

where $B_{c}$ denotes the critical value of the parameter $B$. The other parameter has the following value at the crisis: $A=-1.5$.

See Appendix C for further details on this map.

Central to the proposed analogy with statistical physics will be the identification of the order-parameter for each system. It will transpire that, in each case, the dynamical descriptor playing this role will be the positive Lyapunov exponent, $\lambda^{+}$.

In accordance with the predictions of the theory of critical phenomena, we shall observe that $\lambda^{+}$scales "near" the critical point as

$$
\left(\lambda^{+}-\lambda_{c}^{+}\right) \sim\left|A-A_{c}\right|^{\beta}
$$

where $\lambda_{c}^{+}$is the value of the positive Lyapunov exponent at the critical point and $\beta$ is the scaling exponent describing how $\lambda^{+}$scales as $A \rightarrow A_{c}$ through the intermittent regime for fixed $B$.

The dynamical systems considered here are all twodimensional mappings with two control parameters, conventionally represented as $A$ and $B$. The main idea put forth in this study is that there are a pair of scaling exponents associated with the scaling of $\lambda^{+}$as $(A, B) \rightarrow$ $\left(A_{c}, B_{c}\right)$ where $\left(A_{c}, B_{c}\right)$ is the set of parameter values at which the critical point occurs. These scaling exponents are denoted by $\beta_{A}$ and $\beta_{B}$. In light of the analogy being made between the crises in these mappings and the critical point from statistical physics, it seems natural to ask if the set of scaling exponents $\gamma, \beta_{A}$ and $\beta_{B}$ satisfy any of the classical scaling exponent relationships.

We show that these scaling exponents satisfy a scaling relation which is similar to Widom's scaling law, $\gamma=\beta(\delta-1)$ which occurs in the standard theory of critical phenomena in statistical physics [9].

\section{Analogy with statistical physics}

We investigate the above two-dimensional mappings which are in a chaotic regime. Each of the systems exhibits a sharp chaos to chaos transition at a critical set of parameter values $\left(A_{c}, B_{c}\right)$. The fractal structures underpinning the dynamics changes from an ordered phase (in each case this is the islands regime) to a disordered phase (the intermittent regime) in the phase space of system (see Appendices $\mathrm{A}, \mathrm{B}$ and $\mathrm{C}$ for detailed pictures of these fractal structures).

We use the example of spontaneous magnetization in a ferromagnetic system as the motivation for constructing a formal analogy between this model and the chaos $\leftrightarrow$ chaos transitions exhibited by the three dynamical systems investigated here. This analogy is supported by our finding that as $(A, B) \rightarrow\left(A_{c}, B_{c}\right)$ through the intermittent regime, the positive lyapunov exponent $\lambda^{+}$depends on both $A$ and $B$ specifically as follows:

$$
\begin{aligned}
& \left(\lambda^{+}-\lambda_{c}^{+}\right) \sim\left|A-A_{c}\right|^{\beta_{A}} \\
& \left(\lambda^{+}-\lambda_{c}^{+}\right) \sim\left|B-B_{c}\right|^{\beta_{B}}
\end{aligned}
$$

where in the first equation $B$ is fixed and $A$ varied; while in the second equation $A$ is fixed and $B$ is varied.

In the case of a ferromagnet, in the absence of an external magnetic field being applied $(H=0)$ as the temperature is lowered, a critical temperature $T_{c}$ is reached, below which the ferromagnetic substance spontaneously magnetizes. The following scaling relations for $M$ (the magnetization) and $H$ (the external magnetic field) hold

$$
\begin{aligned}
& M \sim\left|T-T_{c}\right|^{\beta} \\
& M \sim H^{1 / \delta}
\end{aligned}
$$

where in the second equation, it is assumed that $T=T_{c}$. The analogy proposed here is that the parameters $A$ and $B$ correspond to the variables $T$ and $H$ and the scaling exponents $\beta_{A}$ and $\beta_{B}$ correspond to $\beta$ and $1 / \delta$.

Intuitively we might expect that for the dynamical systems it would not make any difference whether $A$ or $B$ corresponds to the thermal variable $T$. When the results are examined it will be suggested that this is in fact the case for the particular maps studied.

\section{Intermittency scaling exponent $\gamma$}

In the crisis considered in this paper the dynamics of the orbit in each case becomes intermittent as the varied parameter, $A$, passes through its critical value. This intermittent behaviour can be characterised, in each case, by an 
Table 1. Intermittency scaling exponent $\gamma$ for the Henon, Mira 1 and Mira 2 maps.

\begin{tabular}{cc}
\hline Mapping & $\gamma$ \\
\hline Henon & $0.51 \pm 0.01$ \\
Mira 1 & $0.52 \pm 0.01$ \\
Mira 2 & $0.505^{*} \pm 0.01$ \\
\hline
\end{tabular}

associated scaling exponent, $\gamma$, where $\gamma$ is defined through the scaling relation

$$
\langle\tau\rangle \sim\left|A-A_{c}\right|^{-\gamma}
$$

(If $B$ is the parameter that is varied, then $A$ is replaced by $B$ in the above equation).

For each of the three systems considered, $\gamma$ was calculated using an automated technique which estimates, for a fixed $A$, a large number of $\tau$-values and hence $\langle\tau\rangle$. This procedure was duplicated over a range of values of the parameter $A$ in the intermittent regime and the exponent $\gamma$ was then estimated from a linear-fit to a log-log plot of the above scaling relationship.

The results in all three cases were consistent with the scaling relation (13). We present in Table 1 a summary of the values for the intermittency scaling exponents that we obtained for each of the three systems considered. See Figures 1 to 6 below.

Shown in the figures below are the corresponding plots of these results for the Henon system, the Mira 1 system, and the Mira 2 system respectively.

${ }^{*}$ Figure 3 showing the results for the Mira 2 map merits some comment. It can be seen that there is a distinctive "bump" or "kink" midway along the plot. Repeated tweaking of the procedure used to generate the data failed to eradicate this effect. Investigation of the time-series corresponding to values of the parameter $A$ near this region confirmed the accuracy of the computational procedure and so it would appear that this is a genuine feature of the dynamics.

Fitting a straight line to the upper-region alone, yields a value of $\gamma=0.49 \pm 0.01$, while fitting a straight line to the data below the "kink" gives $\gamma=0.52 \pm 0.01$. Inspection of the local slope plot, Figure $3 \mathrm{~b}$, for the data set indicates a value of $\gamma$ of 0.50 which holds true over six decades except for the region around the kink. Hence we conclude that the true value of $\gamma$ lies somewhere between 0.49 , the slope of the line above the kink, and 0.52 , the slope of the line below the kink. Taking the average of these values gives 0.505 . (The values of $\gamma$ above and below the kink fall within this value to within the error bars. The extension of the anomalous region tends to give an over estimate for the value of $\gamma$ below the kink).

\section{Lyapunov exponent scaling exponents $\beta_{A}$ and $\beta_{B}$}

In order to justify using a statistical physics analogy it was necessary to find some measureable, or system descriptor,

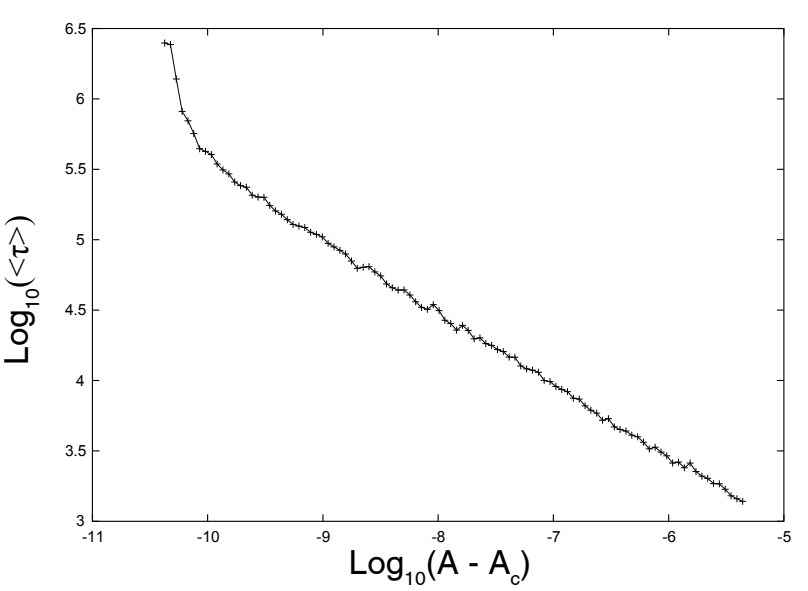

(a)

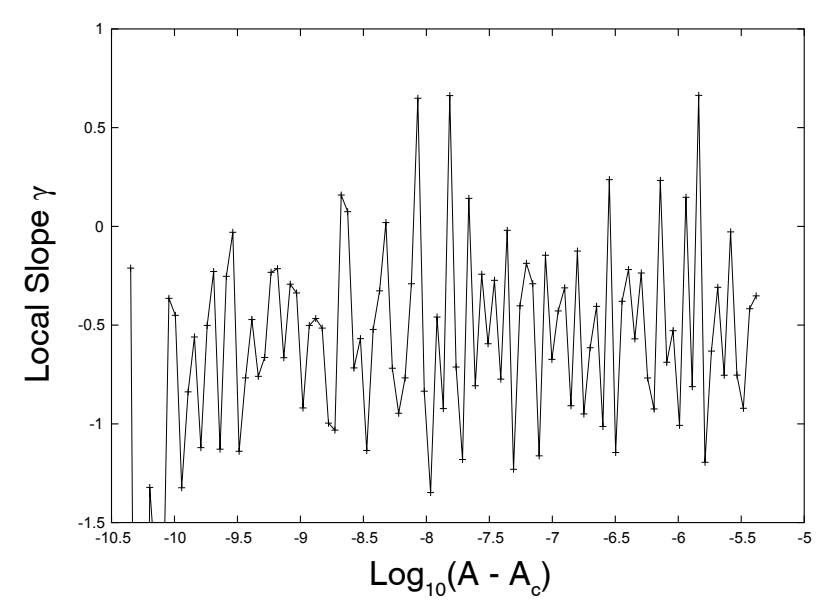

(b)

Fig. 1. The Henon map. (a) Graph of the log of intermittency mean residence time, $\log _{10}(\langle\tau\rangle)$, as a function of $\log _{10}\left(A-A_{c}\right)$ for the Henon map at crisis. (b) Local value of slope $\gamma$ (intermittency scaling exponent) taken two points at a time versus $\log _{10}\left(A-A_{c}\right)$ for the Henon map.

which could be interpreted as a suitable order parameter. The order parameter, in the language of statistical physics, is zero in the ordered phase of the dynamics $\left(A<A_{c}\right)$, and non-zero for $A>A_{c}$, the so-called disordered phase. As $A$ decreases with $A \rightarrow A_{c}$, the order parameter varies continuously but its derivative diverges at $A_{c}$. The descriptor which is found to assume the role of order parameter is the positive Lyapunov exponent, $\lambda^{+}$. It should be noted that each of the dynamical systems used in this study are in the chaotic regime and $\lambda^{+}$exhibits order-parameter-like behaviour as the parameter $A$ passes through $A_{c}$. This generic behaviour is shown in Figure $4 \mathrm{a}$ and in Figure $4 \mathrm{~b}$. The abrupt change in the value of $\lambda^{+}$through $A_{c}$ is evident from these results, which is phase transition-like in its variation. It would seem reasonable to conjecture then that $\lambda^{+}$might obey a scaling relation of the form

$$
\left(\lambda^{+}-\lambda_{c}^{+}\right) \sim\left|A-A_{c}\right|^{\beta}
$$




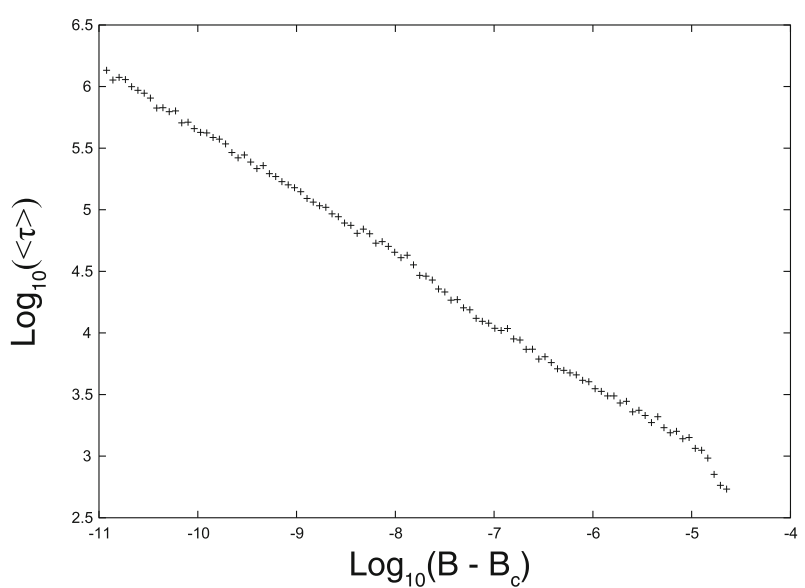

(a)

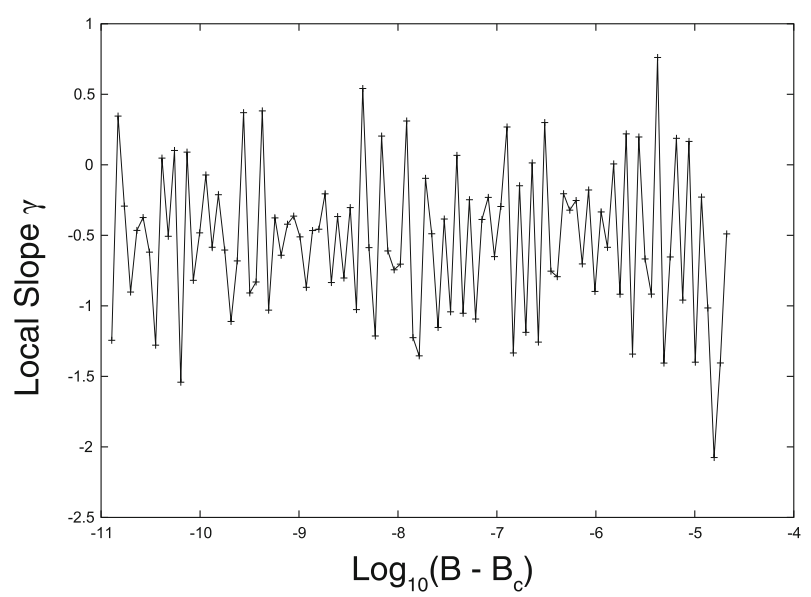

(b)

Fig. 2. The Mira 1 map. (a) Graph of $\log _{10}(\langle\tau\rangle)$ versus $\log _{10}\left(B-B_{c}\right)$ for the Mira 1 map at crisis. (b) Local value of the slope $\gamma$ (intermittency scaling exponent) taken two points at a time versus $\log _{10}\left(B-B_{c}\right)$ for the Mira 1 Map.

In making an analogy with statistical physics, the varied parameter (which is generically called $A$ ) is to be interpreted as a thermal variable and it is with its variation, that the dynamics of each system changes. The claim of this study is that there is a pair of scaling exponents, labelled $\beta_{A}$ and $\beta_{B}$, which arise out of proposed separate scaling relations that describe the variation of $\lambda^{+}$as $(A, B) \rightarrow\left(A_{c}, B_{c}\right)$. It should be noted that each of the three systems are two-parameter dynamical systems, with the labels $A$ and $B$ defining these parameters.

Note that $\lambda_{c}^{+}$is the value of $\lambda^{+}$at $\left(A_{c}, B_{c}\right)$, where $\left(A_{c}, B_{c}\right)$ is the location in parameter-space of the critical point.

More specifically, the scaling relations in question have the form

$$
\begin{aligned}
& \left(\lambda^{+}-\lambda_{c}^{+}\right) \sim\left|A-A_{c}\right|^{\beta_{A}} \\
& \left(\lambda^{+}-\lambda_{c}^{+}\right) \sim\left|B-B_{c}\right|^{\beta_{B}}
\end{aligned}
$$

where the first relation is at fixed $B$ and the second is at fixed $A$. Although it may transpire that $\beta_{A}=\beta_{B}$, in gen-

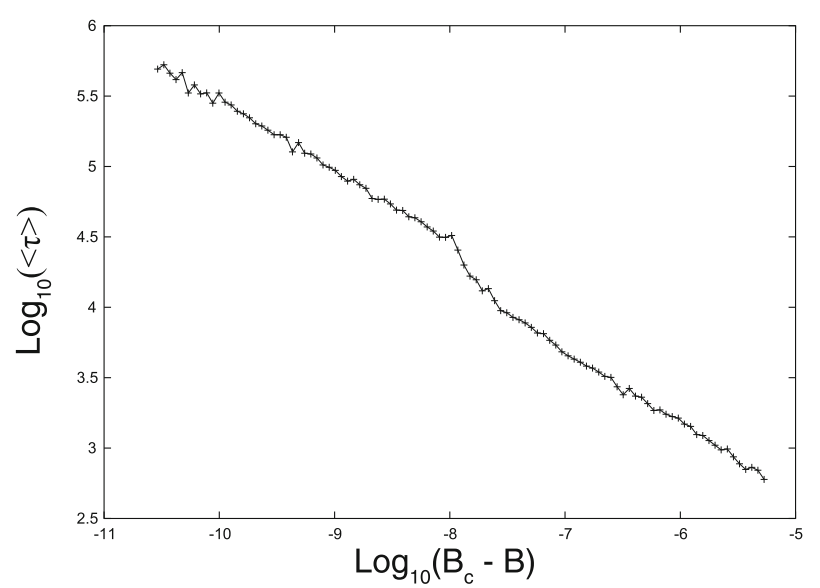

(a)

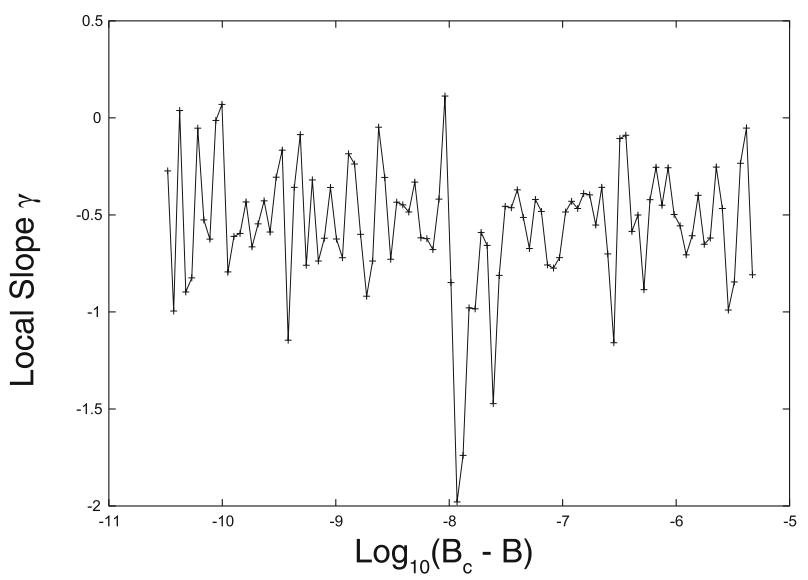

(b)

Fig. 3. The Mira 2 map. (a) Graph of $\log _{10}(\langle\tau\rangle)$ versus $\log _{10}\left(B-B_{c}\right)$ for the Mira 2 map at crisis. (b) Local value of the slope $\gamma$ (intermittency scaling exponent) taken two points at a time versus $\log _{10}\left(B-B_{c}\right)$ for the Mira 2 map.

eral we assume that $\beta_{A} \neq \beta_{B}$. Note that, as in statistical physics, scaling relations like (14) and (15) are generally only first-order approximations and their region of validity diminishes as $\left|A-A_{c}\right|$ (or $\left.\left|B-B_{c}\right|\right)$ increases. Therefore it is to be expected that (14) and (15) will only hold for $A$ (or $B$ ) very "close" to $A_{c}$ (or $B_{c}$ ).

This assumption is reasonable if we persist in making an analogy with statistical physics where, for instance, in the case of spontaneous magnetization, the scaling exponents describing the scaling of the magnetization $M$ below the critical temperature $T_{c}$ are assumed to be different. In that example the exponents corresponding to $\beta_{A}$ and $\beta_{B}$ are labelled $\frac{1}{\delta}$ and $\beta$ and are defined through the scaling relations

$$
\begin{aligned}
& M \sim\left|T-T_{c}\right|^{\beta} \\
& M \sim H^{1 / \delta}
\end{aligned}
$$

where in the second relation it is assumed that $T=T_{c}$, and $H$ is the external magnetic field. 


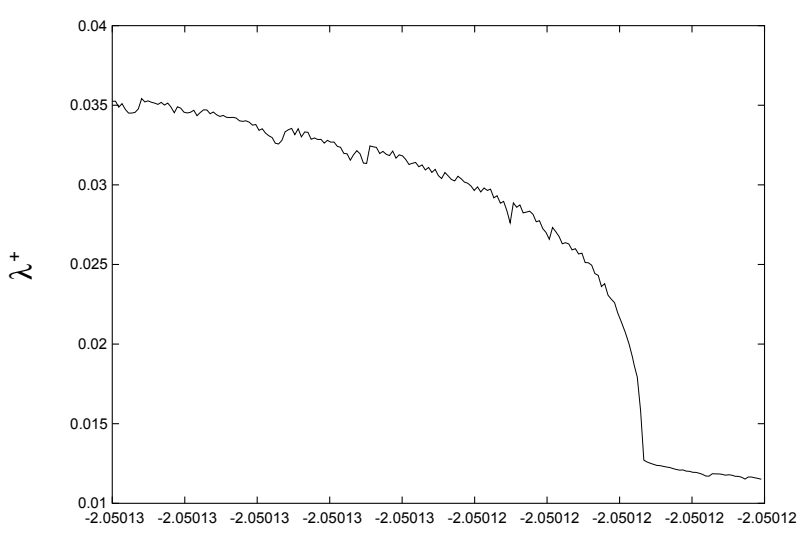

B

(a)

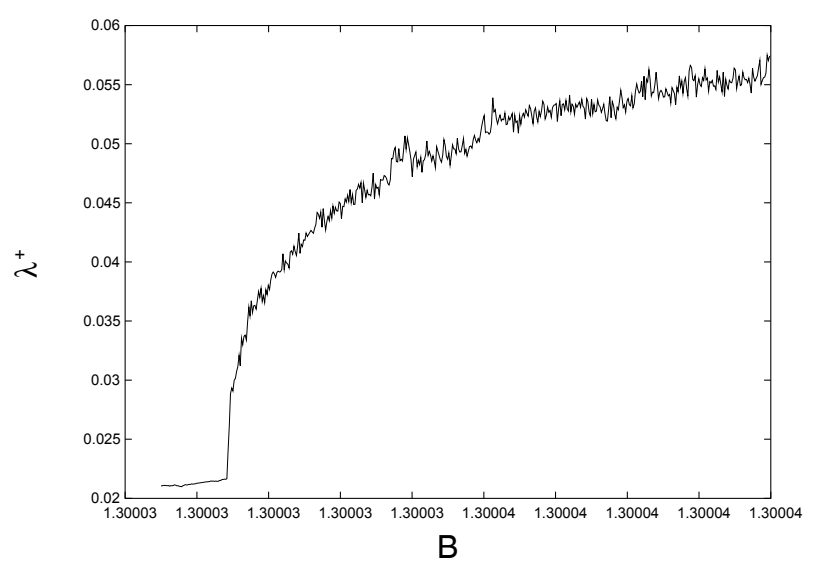

(b)

Fig. 4. Variation of the order parameter through the crisis for the Mira 2 and Mira 1 maps. (a) Variation of $\lambda^{+}$as $B$ passes through $B_{c}$ : Mira 2 map. (b) Variation of $\lambda^{+}$as $B$ passes through $B_{c}$ : Mira 1 map.

Table 2. The Lyapunov scaling exponents $\beta_{A}$ and $\beta_{B}$ for the Henon, Mira 1 and Mira 2 maps.

\begin{tabular}{ccc}
\hline Mapping & $\beta_{A}$ & $\beta_{B}$ \\
\hline Henon & $0.497 \pm 0.005$ & $0.503 \pm 0.005$ \\
Mira 1 & $0.490 \pm 0.003$ & $0.502 \pm 0.002$ \\
Mira 2 & $0.50 \pm 0.01$ & $0.51 \pm 0.01$ \\
\hline
\end{tabular}

Indeed we find this assumed scaling for the three dynamical systems considered and we calculate a pair of scaling exponents, $\beta_{A}$ and $\beta_{B}$ for each system. The results are summarized in Table 2 and the individual plots of the data are shown in the figures from Figures 5 to 10 .

It can be seen from the graphs of the resulting data that in all cases there is a well defined linear scaling region, extending over between three and five decades. We conclude that the proposed scaling relations involving $\beta_{A}$ and $\beta_{B}$ are valid, at least over a significant range of parameter values. The apparent degradation of the linear region in all cases, for "small" $\left|A-A_{c}\right|$, can be attributed to the lack of sufficient accuracy in the calculated value for $\lambda_{c}^{+}$. This is because the computation required to satisfactorily

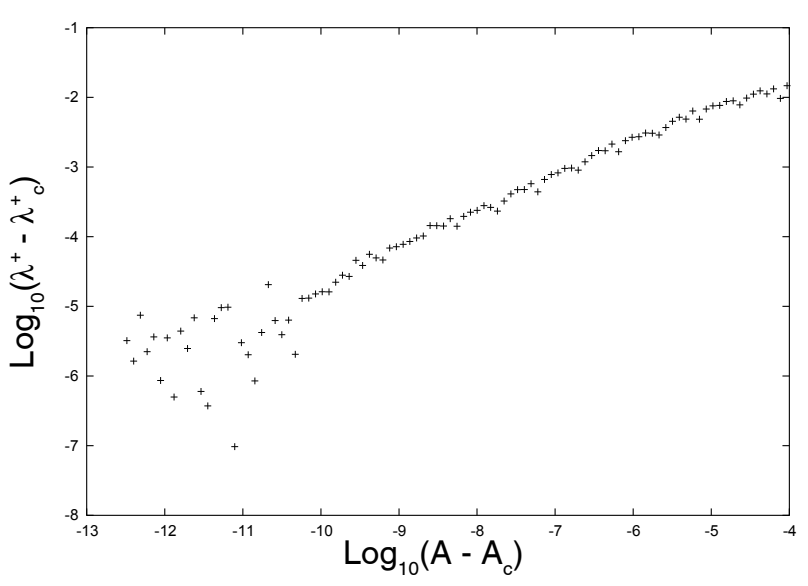

(a)

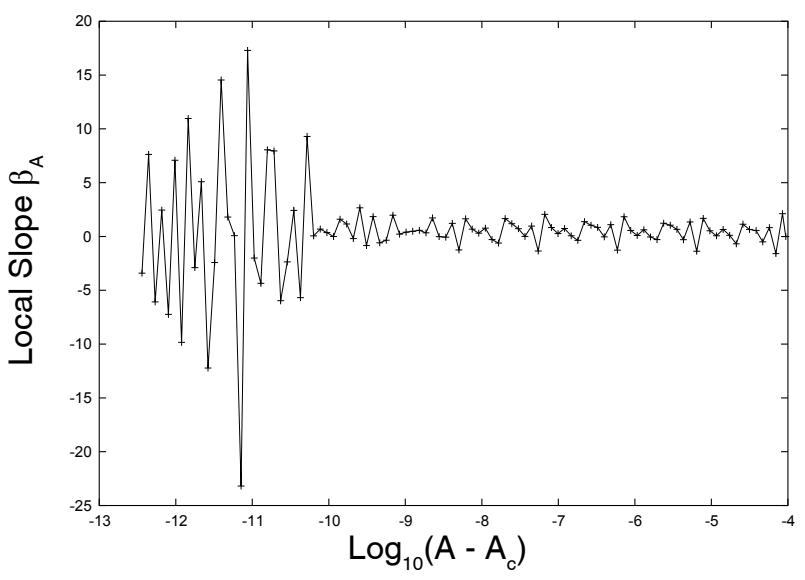

(b)

Fig. 5. Obtaining the Lyapunov scaling exponent, $\beta_{A}$, for the Henon map. (a) Graph of the $\log _{10}\left(\lambda^{+}-\lambda_{c}^{+}\right)$versus $\log _{10}\left(A-A_{c}\right)$, the slope of which yields $\beta_{A}$. (b) The local slope analysis of the log-log plot of (a) above.

calculate both $\lambda_{c}^{+}$and $\lambda^{+}$, as $A$ approaches $A_{c}$, becomes prohibitive. In essence, as $A$ approaches $A_{c}$, we need to estimate $\lambda_{c}^{+}$and $\lambda^{+}$with greater and greater accuracy to continue to see the linear scaling already observed. Both $\lambda_{c}^{+}$and $\lambda^{+}$have been calculated using orbits of length $10^{7}$ and each has been averaged over 100 initial points. These calculations required significant computer time but were sufficient to reveal the proposed scaling of $\lambda^{+}$with $A$ (or $B)$.

Examination of the table of results indicates that, possibly, $\beta_{A}=\beta_{B}=1 / 2$.

One other point to note is that, in relation to the Henon map, the value of $\beta_{A}$ found here is in conflict with the value quoted by Mehra and Ramaswamy [10] where they find that $\beta_{A}=0.37$. In their calculations they used orbits of length $10^{4}$ and averaged over 50 different initial conditions. It is not stated there how accurately $\lambda_{c}$ was calculated but it can probably be assumed that it was estimated using the integrating scheme with similar orbit lengths. We found in our calculations that the accuracy of the results for $\beta_{A}$ and $\beta_{B}$ were very sensitive to the 


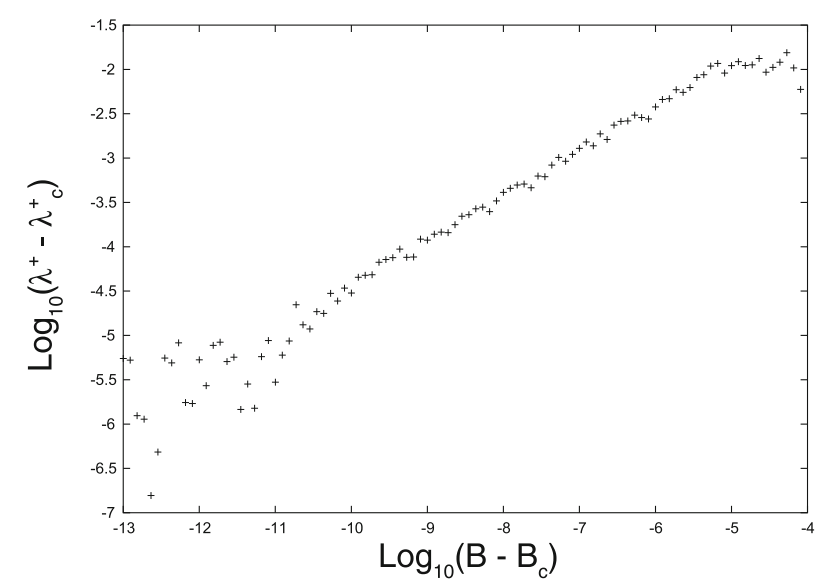

(a)

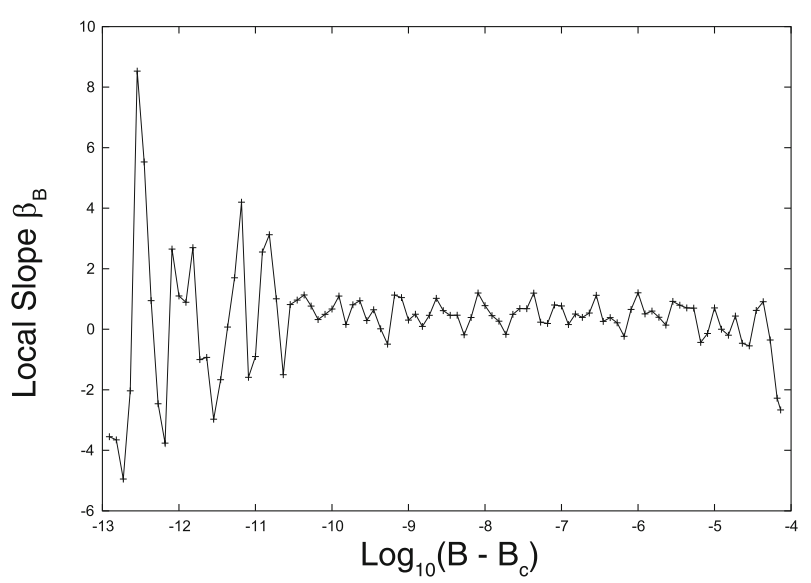

(b)

Fig. 6. Obtaining the Lyapunov scaling exponent, $\beta_{B}$, for the Henon map. (a) Graph of the $\log _{10}\left(\lambda^{+}-\lambda_{c}^{+}\right)$versus $\log _{10}\left(B-B_{c}\right)$, the slope of which yields $\beta_{B}$. (b) The local slope analysis of the log-log plot of (a) above.

value of $\lambda_{c}$ used. The use of longer orbits and bigger sets of initial conditions allows us to better estimate $\lambda_{c}$ and thus achieve better accuracy in calculating the critical exponents.

\section{Discussion and conclusions}

The central claim here is that the scaling exponents $\gamma, \beta_{A}$ and $\beta_{B}$ satisfy a scaling law analogous to Widom's scaling law

$$
\gamma=\beta(\delta-1) .
$$

In the case studied here, where a formal analogy with the spontaneous magnetization example of statistical physics is proposed,

$$
\begin{aligned}
& \gamma \rightarrow \gamma \\
& \beta \rightarrow \beta_{B} \\
& \delta \rightarrow \frac{1}{\beta_{A}}
\end{aligned}
$$

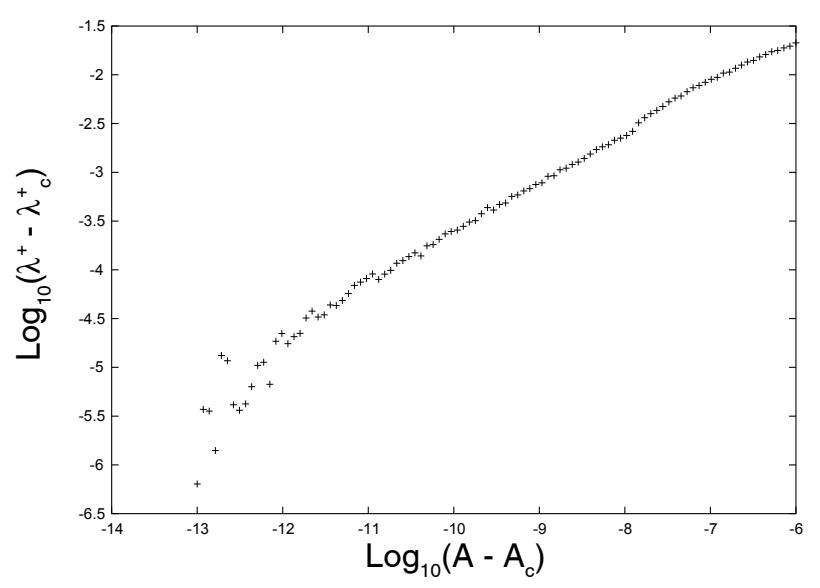

(a)

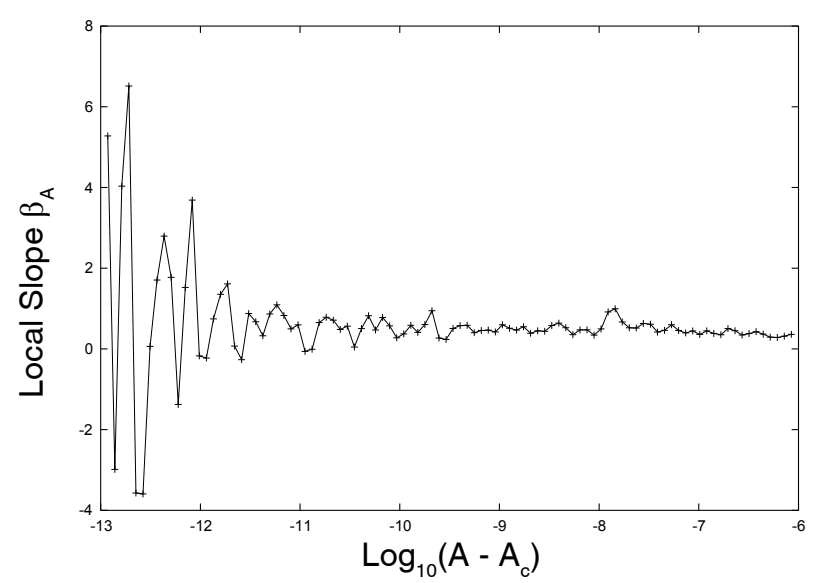

(b)

Fig. 7. Obtaining the Lyapunov scaling exponent, $\beta_{A}$, for the Mira 1 map. (a) Graph of the $\log _{10}\left(\lambda^{+}-\lambda_{c}^{+}\right)$versus $\log _{10}\left(A-A_{c}\right)$, the slope of which yields $\beta_{A}$. (b) The local slope analysis of $\beta_{A}$ taken 3 points at a time.

Table 3. $\gamma_{\text {calc }}$ and $\gamma_{\text {pred }}=\beta_{B}\left(\frac{1}{\beta_{A}}-1\right)$ for the Henon, Mira 1 and Mira 2 maps.

\begin{tabular}{ccc}
\hline Mapping & $\gamma_{\text {calc }}$ & $\gamma_{\text {pred }}=\beta_{B}\left(\frac{1}{\beta_{A}}-1\right)$ \\
\hline Henon & $0.51 \pm 0.01$ & 0.51 \\
Mira 1 & $0.52 \pm 0.01$ & 0.52 \\
Mira 2 & $0.50 \pm 0.01$ & 0.50 \\
\hline
\end{tabular}

the modified form for this scaling law then is

$$
\gamma=\beta_{B}\left(\frac{1}{\beta_{A}}-1\right)
$$

Table 3 presents a summary of results based on this assumed scaling. $\gamma_{\text {calc }}$ is the calculated value for the intermittency scaling exponent and $\gamma_{\text {pred }}$ is the value of $\gamma$ predicted by equation (22). There is excellent agreement between $\gamma_{\text {calc }}$ and $\gamma_{\text {pred }}$.

If we assume that neither parameter has any greater importance than the other then we are forced to consider 


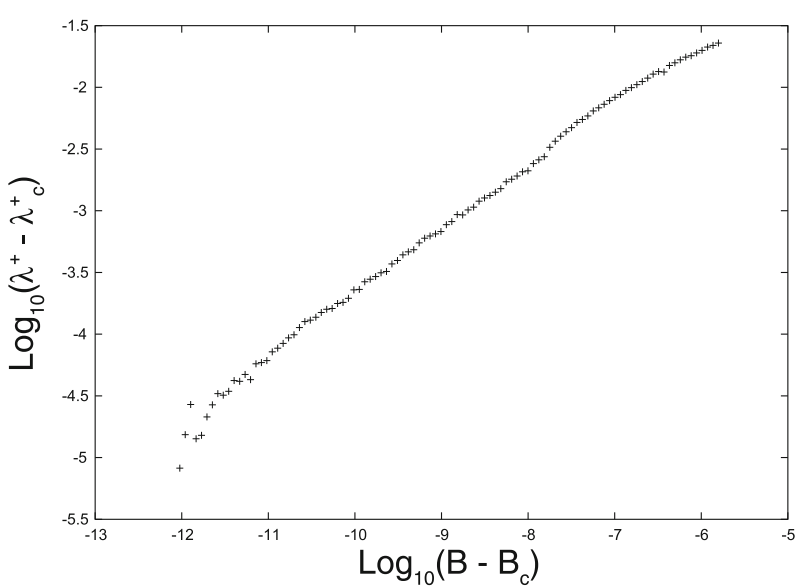

(a)

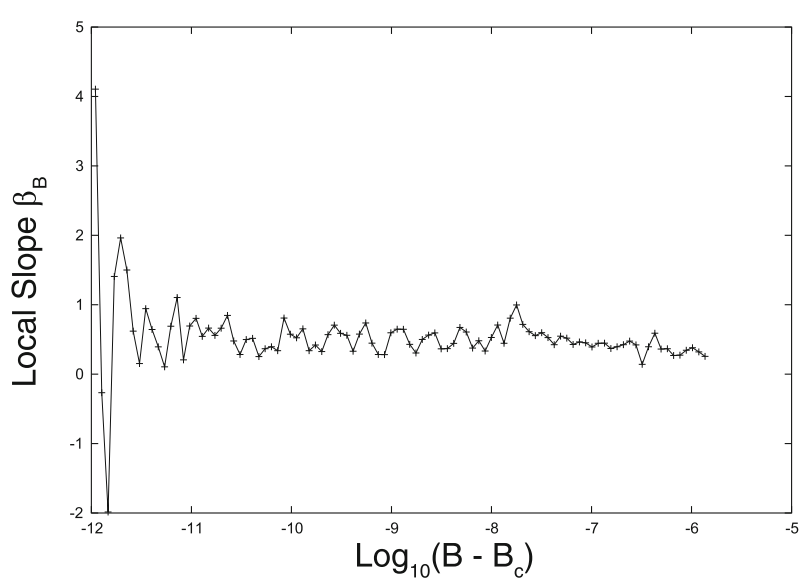

(b)

Fig. 8. Obtaining the Lyapunov scaling exponent, $\beta_{B}$, for the Mira 1 map. (a) Graph of the $\log _{10}\left(\lambda^{+}-\lambda_{c}^{+}\right)$versus $\log _{10}\left(B-B_{c}\right)$, the slope of which yields $\beta_{B}$. (b) The local slope analysis of $\beta_{B}$ taken 3 points at a time.

the possibility that the formal analogy with statistical physics might take the form

$$
\begin{aligned}
& \gamma \rightarrow \gamma \\
& \beta \rightarrow \beta_{A} \\
& \delta \rightarrow \frac{1}{\beta_{B}} .
\end{aligned}
$$

In this case, (22) becomes

$$
\gamma=\beta_{A}\left(\frac{1}{\beta_{B}}-1\right)
$$

If this is the case we obtain the results summarized in Table 4 . While agreement is less impressive, when the associated errors are taken into account then an equation having the form of equation (18) still appears to hold.

The results obtained for the scaling exponents $\beta_{A}$ and $\beta_{B}$ in Table 2 suggest that for the particular mappings

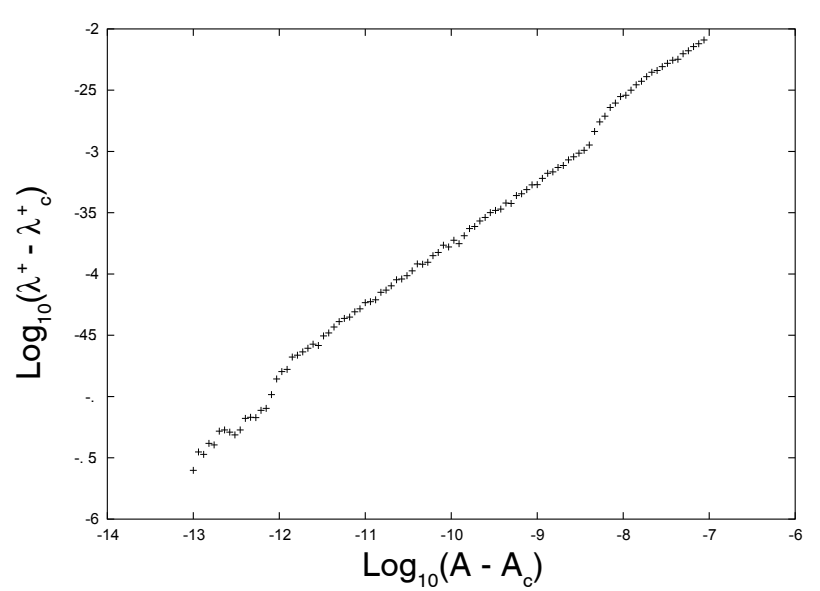

(a)

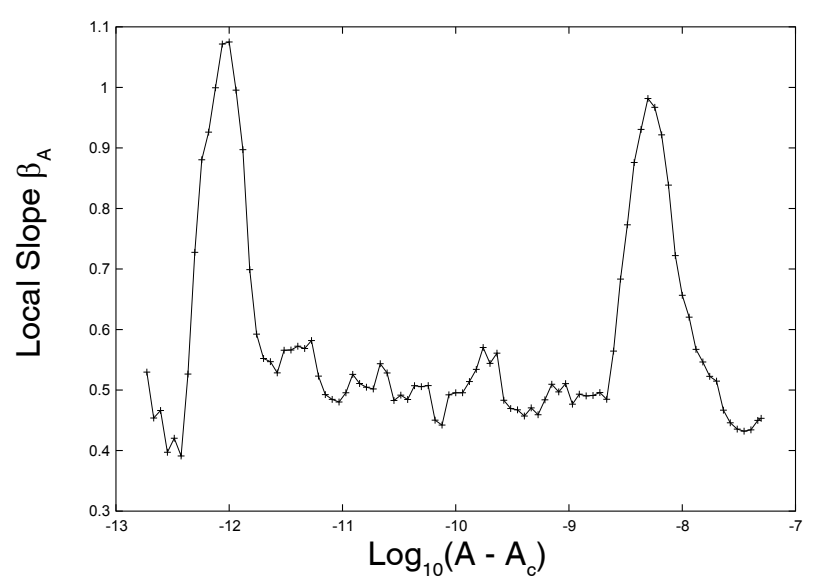

(b)

Fig. 9. Obtaining the Lyapunov scaling exponent, $\beta_{A}$, for the Mira 2 map. (a) Graph of the $\log _{10}\left(\lambda^{+}-\lambda_{c}^{+}\right)$versus $\log _{10}\left(A-A_{c}\right)$, the slope of which yields $\beta_{A}$. (b) The local slope analysis of $\beta_{A}$ taken 10 points at a time.

Table 4. $\gamma_{\text {calc }}$ and $\gamma_{\text {pred }}=\beta_{A}\left(\frac{1}{\beta_{B}}-1\right)$ for the Henon, Mira 1 and Mira 2 maps.

\begin{tabular}{ccc}
\hline Mapping & $\gamma_{\text {calc }}$ & $\gamma_{\text {pred }}=\beta_{A}\left(\frac{1}{\beta_{B}}-1\right)$ \\
\hline Henon & $0.51 \pm 0.01$ & 0.49 \\
Mira 1 & $0.52 \pm 0.01$ & 0.49 \\
Mira 2 & $0.50 \pm 0.01$ & 0.49 \\
\hline
\end{tabular}

which we have studied $\beta_{A}=\beta_{B}=\beta$. If this is true then equation $(22)$ reduces to

$$
\gamma=1-\beta
$$

The numerical results above also suggest that $\beta_{A}=\beta_{B}=$ $\frac{1}{2}$. Using equation (27) this implies that in all cases $\gamma=\frac{1}{2}$ in agreement with our numerical results. It would appear that these results are true for all maps with quadratic nonlinearities.

Grebogi et al. [4] assert that for all one-dimensional maps with quadratic extrema, $\gamma=\frac{1}{2}$. While all of the examples considered here are two-dimensional maps, they 


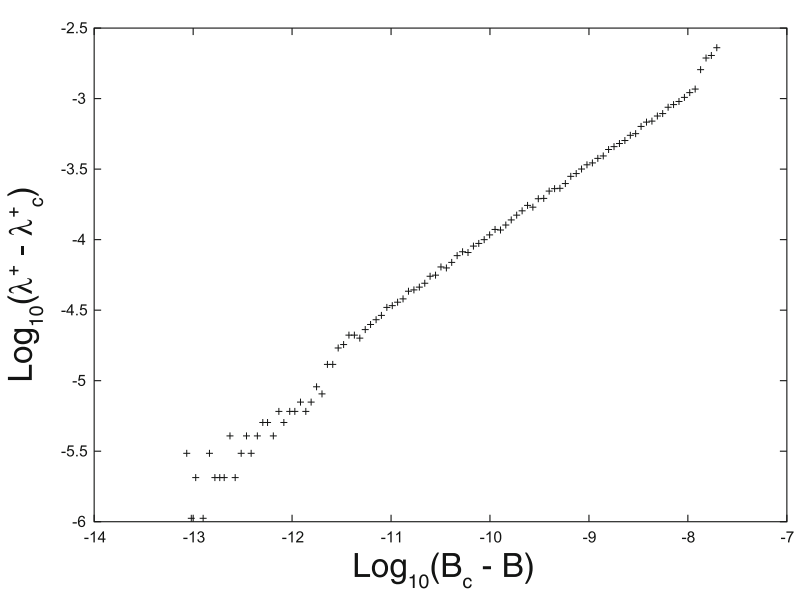

(a)

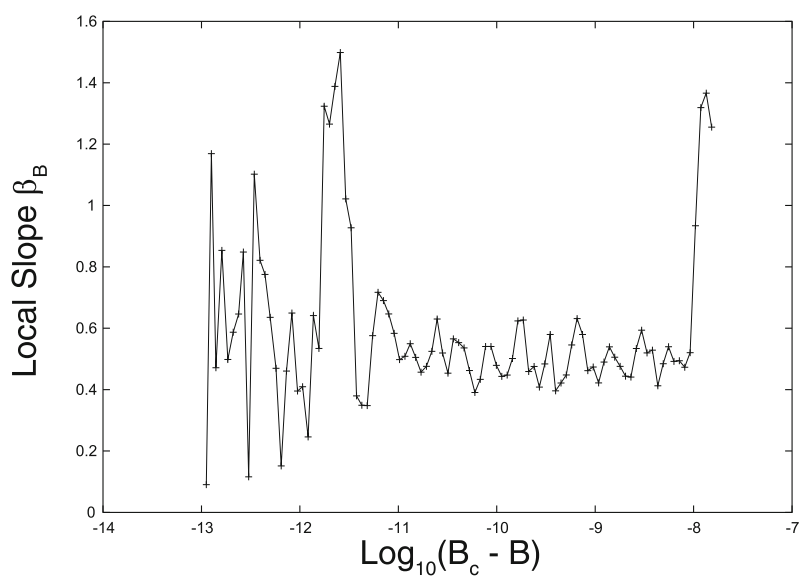

(b)

Fig. 10. Obtaining the Lyapunov scaling exponent, $\beta_{B}$, for the Mira 2 map. (a) Graph of the $\log _{10}\left(\lambda^{+}-\lambda_{c}^{+}\right)$versus $\log _{10}\left(B-B_{c}\right)$, the slope of which yields $\beta_{B}$. (b) The local slope analysis of $\beta_{B}$ taken 5 points at a time.

all have a quadratic functional form in either $X_{n+1}$ or $Y_{n+1}$. Inspection of the attractors for both regimes $A<A_{c}$ and $A>A_{c}$, for the three dynamical systems considered, indicate fractal line-like structure in the phase space. In light of this fact, the results would not be totally unexpected. This hypothesis could be tested by considering two-dimensional systems

$$
\begin{gathered}
X_{n+1}=f_{1}\left(X_{n}, Y_{n} ; A, B\right) \\
Y_{n+1}=f_{2}\left(X_{n}, Y_{n} ; A, B\right)
\end{gathered}
$$

where either $f_{1}\left(X_{n}, Y_{n} ; A, B\right)$ or $f_{2}\left(X_{n}, Y_{n} ; A, B\right)$, or both, had a non-quadratic extrema or a non-quadratic functional form. It might be possible to construct a mapping which has the requisite properties and determine if the same, or different, scaling relations still hold. The Ikeda map might serve as a suitable test map, given that it has a non-quadratic functional form for both $f_{1}\left(X_{n}, Y_{n} ; A, B\right)$ and $f_{2}\left(X_{n}, Y_{n} ; A, B\right)$.
What the results do seem to support is the claim that, for the two-parameter mappings which we have studied, relation (18) seems to be true, and that $\beta_{A}=\beta_{B}=\frac{1}{2}=\gamma$.

In any event, the results suggest that further work may be needed before we can assert that (18) holds for all twodimensional mappings which undergo crisis-induced intermittency.

Note that in all the examples that we have considered we have a chaos-to-chaos transition. This transition is manifested by a fractal strange attractor structure changing to another fractal strange attractor structure as we go through the critical point. In general we would expect that if the strange attractors in phase space at the critical point do not have a line like structure then $\gamma \neq \frac{1}{2}[11], \beta_{A} \neq \beta_{B}$ and equation (22) (or Eq. (26)) is the appropriate relation to use.

This work was partially supported by Science Foundation Ireland's Research Frontiers Programme under Grant PHYF791. We would like to thank Dr. Graham. Kells for all his help and assistance with preparing the paper for publication.

\section{Appendix A: The henon map}

The Henon map is a non-linear two-dimensional mapping which has the following functional form

$$
\begin{aligned}
X_{n+1} & =1+Y_{n}-A X_{n}^{2} \\
Y_{n+1} & =B X_{n} .
\end{aligned}
$$

A crisis is observed to occur when the parameter $A$ falls in the following interval

$$
A_{c} \in[1.2716856034576725,1.2716856034576735]
$$

where $A_{c}$ denotes the critical value of the parameter $A$. The other parameter has the following values at the crisis: $B=0.3$.

Hence, the critical point occurs at $\left(A_{c}, B_{c}\right)=$ $(1.2716856034576725,0.3)$. The value of the positive lyapunov exponent at the critical point, $\lambda_{c}^{+}$, is

$$
\lambda_{c}^{+}=0.1206471485 \ldots
$$

The initial values of both $X$ and $Y$ were chosen to be $X_{0}=$ 0.5 and $Y_{0}=0.5$. The attractors are shown in Figure 11 for $A<A_{c}$ and $A>A_{c}$.

For $A<A_{c}$ the attractor consists of seven separate pieces or islands which are geometrically similar. i.e they share the same fractal structure. An orbit on this attractor moves from one piece to the next in a cyclic fashion, although the dynamics on an individual island is chaotic. An attractor for $A<A_{c}$ is shown in Figure 11a.

As the parameter $A$ passes through the critical value, $A_{c}$, the system undergoes a crisis and the attractor suddenly enlarges in phase space volume. This can be seen graphically in Figure 11b. 


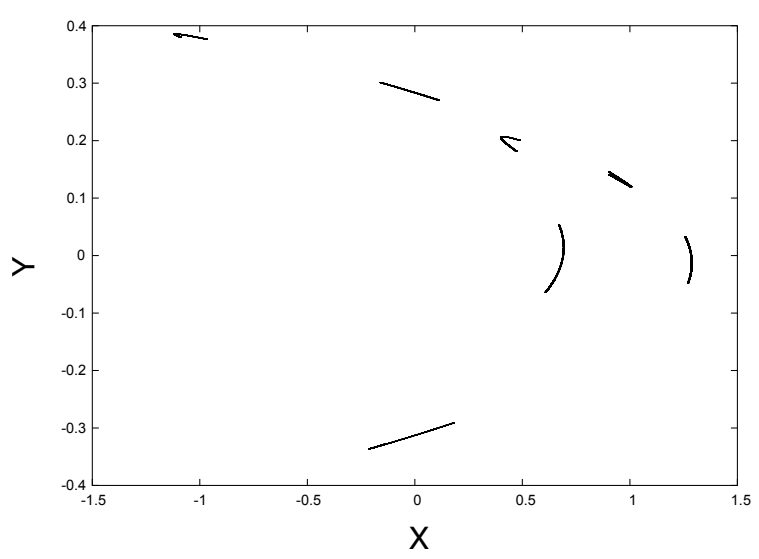

(a)

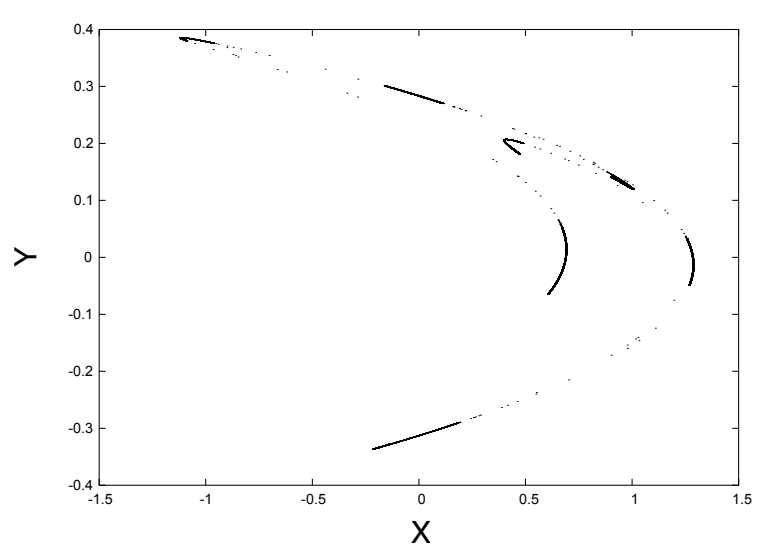

(b)

Fig. 11. Henon map before and after crisis. (a) $A=1.271685$. (b) $A=1.271686$.

This type of dynamics is characterized, for $A$ "close" to $A_{c}$, by intermittent behaviour of an orbit. In this instance the orbit intermittently switches between pre-crisis behaviour where it spends some time on the attractor which existed before the crisis and then suddenly bursts out of this region and wanders around on the enlarged attractor. This intermittent behaviour continues ad infinitum.

\section{Appendix B: The first mira map}

The first Mira map [8] has the functional form

$$
\begin{aligned}
X_{n+1} & =Y_{n} \\
Y_{n+1} & =A Y_{n}-X_{n}^{2}+B .
\end{aligned}
$$

A crisis is observed to occur when the parameter, $B$, falls in the following interval

$$
B_{c} \in[1.3000324223680495,1.3000324223680502]
$$

where $B_{c}$ denotes the critical value of the parameter $B$. The other parameter has the following value at the crisis: $A=0.1$. Hence, the critical point occurs at $\left(A_{c}, B_{c}\right)=$

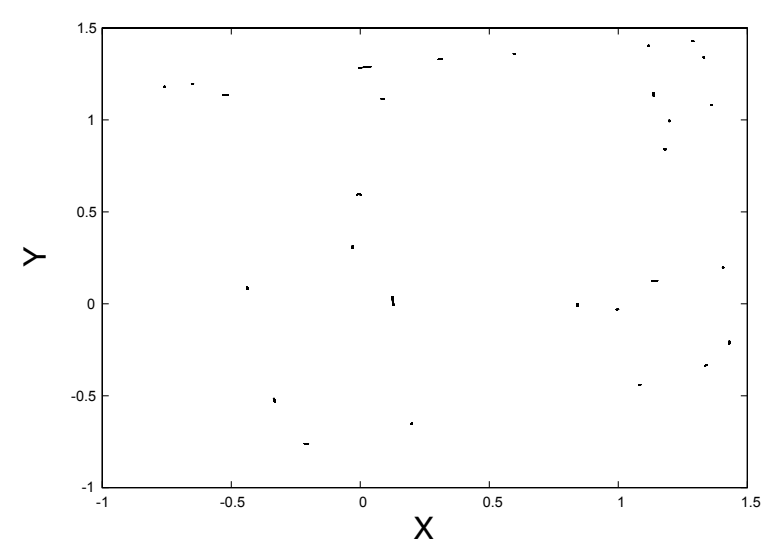

(a)

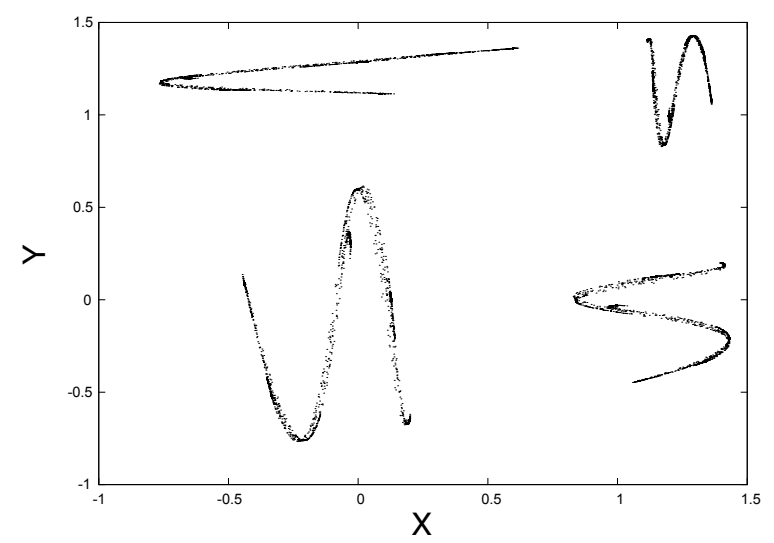

(b)

Fig. 12. Mira 1 map before and after crisis. (a) $B=1.3000324$. (b) $B=1.3000325$.

$(0.1,1.3000324223680495)$. The value of the positive lyapunov exponent at the critical point, $\lambda_{c}^{+}$, is

$$
\lambda_{c}^{+}=0.021702361537 \ldots
$$

The initial values of both $\mathrm{X}$ and $\mathrm{Y}$ were chosen to be $X_{0}=$ 1.0 and $Y_{0}=0.0$. Shown in Figure 12 are the attractors before and after the crisis.

For $B<B_{c}$ the attractor consists of 28 islands, each of which has a similar fractal structure. In this regime an orbit cylces periodically through each of the 28 islands and in this sense the dynamics is periodic although the dynamics on each of the individual islands is chaotic.

For $B>B_{c}$ the attractor has expanded in phase-space volume and the resulting dynamics is intermittent.

\section{Appendix C: The second mira map}

The first Mira map [8] has the functional form

$$
\begin{aligned}
X_{n+1} & =A X_{n}+Y_{n} \\
Y_{n+1} & =X_{n}^{2}+B .
\end{aligned}
$$

A crisis is observed to occur when the parameter, $B$, falls in the following interval

$$
B_{c} \in[-2.05012267960083,-2.05012267960082]
$$




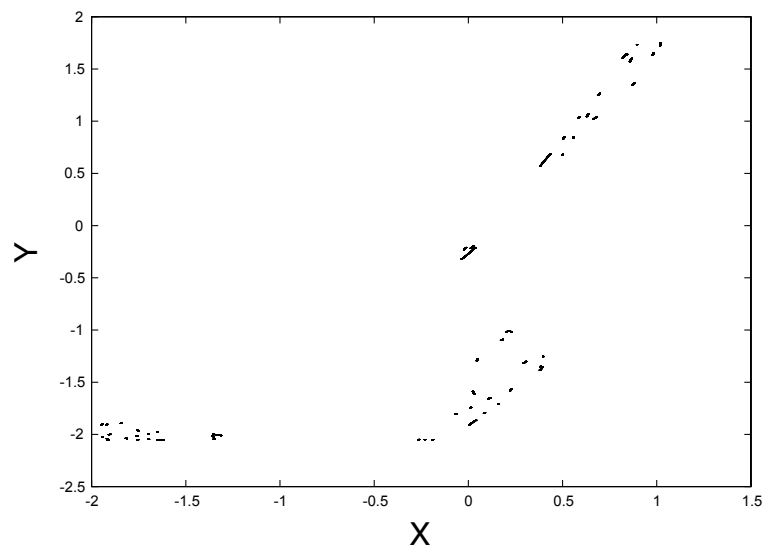

(a)

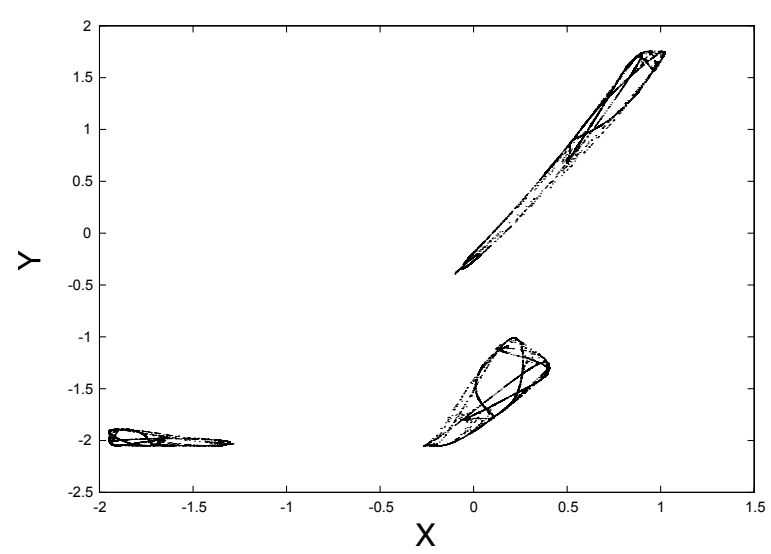

(b)

Fig. 13. Mira 2 map before and after crisis. (a) $B=-2.050122$ : before crisis. (b) $B=-2.050123$ : after crisis.

where $B_{c}$ denotes the critical value of the parameter $B$. The other parameter has the following value at the crisis: $A=-1.5$. Hence, the critical point occurs at

$$
\left(A_{c}, B_{c}\right)=(-1.5,-2.05012267960083) .
$$

The value of the positive lyapunov exponent at the critical point, $\lambda_{c}^{+}$, is

$$
\lambda_{c}^{+}=0.01278943052 \ldots
$$

The initial values of both $\mathrm{X}$ and $\mathrm{Y}$ were chosen to be $X_{0}=0.1$ and $Y_{0}=0.1$. Plots for attractors before and after the crisis are shown in Figure 13.

When $B>B_{c}$ the attractor consists of 108 separate and geometrically similar islands. Again, as with the previous two mappings, an orbit on this attractor hops periodically among these 108 attractors, although the dynamics on each of the individual islands is chaotic.

As $B$ decreases and passes through $B_{c}$ a crisis occurs and the dynamics becomes intermittent, with periods of time spent in the pre-crisis region interspersed with bursts out of this region and into the expanded phase space containing the post-crisis attractor.

\section{References}

1. E. Ott, C. Grebogi, J.A. Yorke, Phys. Rev. A 36, 5365 (1987)

2. H.L. Yang, Z.Q. Huang, E.J. Ding, Phys. Rev. E 55, 6598 (1997)

3. J.C. Sommerer, C. Grebogi, Int. J. Bifur. Chaos 2, 383 (1992)

4. C. Grebogi, E. Ott, Physica D 7, 181 (1983)

5. H.J.H. Clercx, C.J.F. van Heijst, D. Molenaar, Z. Yin. Phys. Rev. E 75, 036309 (2007)

6. E.L. Rempe, F.A. Borott, T. Hada, A.C.L. Chain, W.M. Santana, Y. Kamide, Nonlinear Processes in Geophysics 14, 17 (2007)

7. M.A.F. Sanjuan, G. Tanaka, K. Aihara, Phys. Rev. E 71, 016219 (2005)

8. A. Barugola, J.C. Cathala, C. Mira, L. Gardini, Chaotic dynamics in two-dimensional noninvertible maps (World Scientific, 1996), vol. 20 of A

9. K. Huang, Statistical Mechanics (Wiley, New York, 1987)

10. V. Mehra, R. Ramaswamy, Phys. Rev. E 53, 3420 (1996)

11. J.A. Yorke, C. Grebogi, E. Ott, Phys. Rev. Lett. 57, 1284 (1986) 